\title{
Precise Control of Cu Nanoparticle Size and Catalytic Activity through Pore Templating in Zr Metal-Organic Frameworks
}

\author{
Mohammad Rasel Mian, ${ }^{\ddagger, 1}$ Louis R. Redfern, ${ }^{\ddagger 1,2}$ Saied Md Pratik, ${ }^{3}$ Debmalya Ray, ${ }^{3}$ Jian Liu, ${ }^{1}$ \\ Karam B. Idrees, ${ }^{1}$ Timur Islamoglu, ${ }^{1}$ Laura Gagliardi, ${ }^{3 *}$ Omar K. Farha ${ }^{1 *}$ \\ ${ }^{1}$ International Institute of Nanotechnology and Department of Chemistry, Northwestern University, 2145 Sheridan \\ Road, Evanston, Illinois 60208, United States. \\ ${ }^{2}$ X-ray Science Division, Advanced Photon Source, Argonne National Laboratory, 9700 South Cass Avenue, Lemont, \\ Illinois 60439, United States. \\ ${ }^{3}$ Department of Chemistry, Chemical Theory Center and Supercomputing Institute, University of Minnesota, 207 \\ Pleasant Street SE, Minneapolis, Minnesota 55455-0431, United States.
}

\section{Table of Contents}

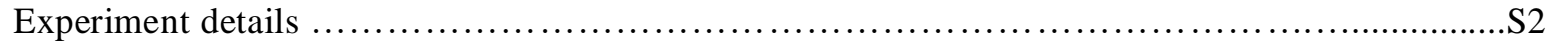

Syntheses of NU-901, Cu-SIM-NU-901, NU-907, Cu-SIM-NU-907 ..........................S4

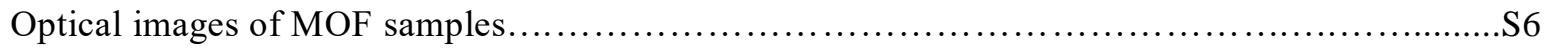

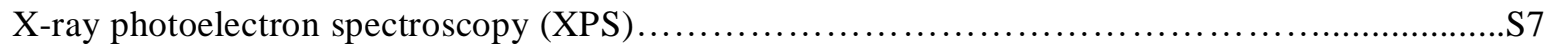

PXRD pattern for NU-901, Cu-SIM-NU-901 and Post catalysis of CuNPs@NU-901 _....................S8

PXRD pattern for NU-907, Cu-SIM-NU-907 and Post catalysis of CuNPs@NU-907.....................S9

$\mathrm{N}_{2}$ isotherm for NU-901, Cu-SIM-NU-901 and Post catalysis of CuNPs@ NU-901........................S10

N2 isotherm NU-907, Cu-SIM-NU-907 and Post catalysis of CuNPs@ @U-907.............................S11

Pore-size distribution of NU-901, Cu-SIM-NU-901 and post-catalysis CuNPs@NU-901...........S12

Pore-size distribution of NU-907, Cu-SIM-NU-907 and post-catalysis CuNPs@NU-907...........S13

SEM image of NU-901, NU-907, Cu-SIM-NU-901 and Cu-SIM-NU-907 ......................S15

Computational methods and models .................................................... 16

Representative Gas Chromatograms.................................................... 18

Diffusion limit test for CuNPs@NU-901_........................................................................................S19

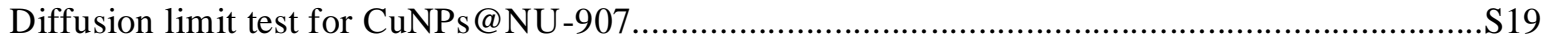

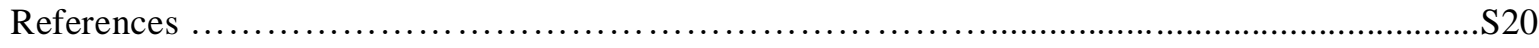




\section{Experiment Details}

\section{Characterization and Instrumentation}

X-ray diffraction analysis: PXRD data were collected at the IMSERC X-ray Facility at Northwestern University on a STOE-STADI-MP powder diffractometer equipped with an asymmetric curved Germanium monochromator $\left(\mathrm{CuK}_{\alpha 1}\right.$ radiation, $\left.\lambda=1.54056 \AA\right)$ and one dimensional silicon strip detector (MYTHEN2 1K from DECTRIS). The line focused Cu Xray tube was operated at $40 \mathrm{kV}$ and $40 \mathrm{~mA}$. Powder was packed in a $3 \mathrm{~mm}$ metallic mask and sandwiched between two layers of polyimide tape. Intensity data from 2 to 30 degrees $2 \theta$ were collected over a period of 10-20 mins. The instrument was calibrated against a NIST Silicon standard (640d) prior the measurement.

Sorption studies: $\mathrm{N}_{2}$ isotherms were measured on Micromeritics Tristar II (Micromeritics, Norcross, GA) instrument at $77 \mathrm{~K}$. Pore-size distributions were obtained using a DFT carbon slit-pore model. Before each run, samples were activated at $120{ }^{\circ} \mathrm{C}$ for NU-901, $150{ }^{\circ} \mathrm{C}$ for NU-907, $65{ }^{\circ} \mathrm{C}$ for $\mathrm{Cu}-\mathrm{SIM}-\mathrm{NU}-901$ and $150{ }^{\circ} \mathrm{C}$ for $\mathrm{Cu}-\mathrm{SIM}-\mathrm{NU}-907$ for 12-24 h under high vacuum on a Smart Vacprep from Micromeritics. Around 30-50 mg of sample was used in each measurement.

Inductively coupled plasma optical emission spectroscopy (ICP-OES): ICP was conducted on an iCAPTM 7600 ICP-AES Analyzer (Thermo Scientific ${ }^{\text {TM}}$ ) over the 166-847 $\mathrm{nm}$ spectral range. Samples (1-3 mg) were digested in a small amount $(1 \mathrm{~mL})$ of a concentrated nitric acid (trace metal grade) by heating in a Biotage (Uppsala, Sweden) SPX microwave reactor (software version 2.3, build 6250) at $150{ }^{\circ} \mathrm{C}$ for 15 minutes or in oil bath at $120{ }^{\circ} \mathrm{C}$ for overnight. $9 \mathrm{~mL}$ Millpore $\mathrm{H} 2 \mathrm{O}$ added to the mother solution to make $10 \mathrm{~mL}$. This solution was analyzed for $\mathrm{Cu}(324.754,327.396$, and $224.700 \mathrm{~nm})$ and $\mathrm{Zr}(327.305$, $339.198,343.823$, and $349.621 \mathrm{~nm}$ ) content as compared to standard solution of 1.563, 3.125, 6.25, 12.5, 25 and $50 \mathrm{ppm}$ of $\mathrm{Zr}$ and $\mathrm{Cu}$.

X-ray photoelectron spectroscopy (XPS): XPS measurements were carried out at the KECK-II/NUANCE facility at Northwestern University on a Thermo Scientific ESCALAB $250 \mathrm{Xi}(\mathrm{Al} \mathrm{K} \alpha$ radiation, $h v=1486.6 \mathrm{eV}$ ) equipped with an electron flood gun. All spectra from XPS were analyzed as reference to the $\mathrm{C} 1 \mathrm{~s}$ peak $(284.8 \mathrm{eV})$ by using Thermo Scientific Advantage Data System software. 
Scanning electron microscopy (SEM): SEM images were taken using a Hitachi S4800 at the EPIC facility (NUANCE Center-Northwestern University). Activated samples were coated with $\mathrm{OsO}_{4}(\sim 9 \mathrm{~nm}$ thickness) in a Denton Desk III TSC Sputter Coater before imaging.

Synchrotron X-ray Experiments: High resolution X-ray diffraction data and total scattering data suitable for difference envelope density (DED) and pair distribution function (PDF) analyses were collected on the 11-ID-B beamline at the Advanced Photon Source at Argonne National Laboratory using $58.7 \mathrm{keV}(\lambda=0.2113 \AA)$ X-rays. Two-dimensional data were collected using a Perkin Elmer amorphous silicon-based area detector. Geometric corrections and reduction to one-dimensional data were performed using GSAS-II. ${ }^{1}$ Samples were loaded into a Kapton capillary in a flow-cell furnace to control temperature and gasphase environment. ${ }^{2}$ Diffraction data for DED analysis were collected with a long $(1 \mathrm{~m})$ sample-to-detector distance to maximize resolution and a data collection time of $10 \mathrm{~s}$. Total scattering data were collected with a shorter sample-to-detector distance $(20 \mathrm{~cm})$ to maximize the $Q$-range obtained with a data collection time of 2 minutes. Data were collected for each sample roughly every 10 minutes to monitor structural changes as a function of time, temperature, and gaseous environment.

For DED analysis, lattice parameters and peak intensities were extracted from highresolution diffraction patterns via Le Bail whole pattern fitting ${ }^{3}$ using Jana2006. ${ }^{4}$ Lattice and pseudo-Voigt profile parameters were refined over a $0.5-10.0^{\circ} 2 \theta$ range. Structure envelopes were generated using the intensities of ten low-index reflections. ${ }^{5}$ Difference envelope densities were calculated by scaling and subtracting the structure envelopes of pristine NU901 and NU-907 from CuNPs@NU-901 and CuNPs@NU-907, respectively, under identical conditions.

For each sample, PDFs were extracted from total scattering data up to $Q_{\max }=21 \AA^{-1}$ using PDFgetX2. ${ }^{6}$ The differential PDF (dPDF) of each sample was calculated by scaling and subtracting the PDF of pristine NU-901 and NU-907 from that of CuNPs@ NU-901 and CuNPs@NU-907, respectively, under identical conditions. Structural models of Cu nanoparticles were refined against the dPDF data using PDFgui $^{7}$ to estimate nanoparticle size. 
Gas-Phase Catalysis: Catalyst activity and selectivity measurements were recorded using a packed-bed flow reactor (Microactivity Efficient, MAE). The gases used for gasphase hydrogenation of acetylene were 5\% acetylene balanced with $\mathrm{Ar}, 5 \% \mathrm{H}_{2}$ balanced with Ar. The catalyst ( 10 mg, diluted with $200-400 \mathrm{mg}$ of high purity, low surface area $\left.\mathrm{SiO}_{2}\right)$ was packed on quartz wool in a stainless-steel reactor. The reaction temperature was controlled with a K-type thermocouple at the top of the catalyst bed. The temperature was ramped at $10{ }^{\circ} \mathrm{C} / \mathrm{min}$ to $200^{\circ} \mathrm{C}$ and held under Ar flow followed by a flow of $10 \mathrm{~mL} / \mathrm{min} \mathrm{H}_{2}$ (5\% diluted with Ar) and $10 \mathrm{~mL} / \mathrm{min}$ ultra-high purity $\operatorname{Ar}(2$ bar total pressure) for 2-4 hours to prepare the $\mathrm{Cu}$ NPs from $\mathrm{Cu}(\mathrm{II})$ species. For catalysis experiments, the ratio of flow rates for acetylene and $\mathrm{H}_{2}$ was held constant at 1:1. Experiments for the determination of turnover frequency were repeated to ensure reproducibility. Further experiments in which the amount of catalyst and flow of all gases were doubled were carried out to ensure that the kinetic data was not diffusion limited. The reactions products were identified as ethylene, 1,3-butadiene and 1-butene by comparing the retention time of authentic standards under identical GC conditions (by using an Agilent 7890A gas GC with FID detector using an Agilent J\&W GC column). The amounts of the gases were determined based on the integration areas converted to $\mathrm{mol} \%$ using relevant calibration curves.

\section{Syntheses of NU-901, Cu-SIM-NU-901, NU-907, Cu-SIM-NU-907}

Zirconyl chloride octahydrate, Zirconium(IV) acetylacetonate, 4-aminobenzoic acid, formic acid, hydrochloric acid, high purity silica, and copper and zirconium ICP standards were purchased from Sigma Aldrich Chemicals Company, Inc. (Milwaukee, WI) and were used as received. Concentrated nitric acid (trace metal grade) was purchased from Fisher Scientific, LLC (Chicago, IL). Bis(dimethylamino-2-propoxy) copper (II) was obtained from Strem Chemicals, and used without further purification. Acetone, chloroform, 1,4-dioxane, N,N dimethylformamide (DMF), and heptane were obtained from Fisher Scientific and used without further purification. Ultrapure deionized water $(18.2 \mathrm{MB} \cdot \mathrm{cm}$ resistivity) was obtained from a Millipore Milli-Q Biocel A10 instrument (Millipore Inc., Billerica, MA). All gases used for the adsorption and desorption measurements were Ultra High Purity Grade 5 and were obtained from Airgas Specialty Gases (Chicago, IL). 
Syntheses of NU-901 and NU-907: NU-901 and NU-907 were synthesized by using $\mathrm{H}_{4}$ TBAPy and $\mathrm{H}_{4}$ abtc linker according to reported procedures. ${ }^{8-9}$

Preparation of Cu-SIM-NU-901: $100 \mathrm{mg} \mathrm{Cu}(\mathrm{dmap})_{2}(0.373 \mathrm{mmol})$ was dissolved in $12 \mathrm{~mL}$ anhydrous heptane under Ar atmosphere in a glovebox. $80 \mathrm{mg}(0.037 \mathrm{mmol})$ activated NU901 were added to this solution and mixture was shaken to disperse the MOF in the solution. A color change from yellow to green was observed. The solution was allowed to stand at room temperature for overnight. The supernatant was removed and replaced with fresh anhydrous heptane $(12 \mathrm{~mL})$. Again the solution was shaken to disperse the MOF and allowed to stand for several hours (around $3 \mathrm{~h}$ ). This process was repeated 2 additional times. The supernatant was replaced with acetone upon removing from the glovebox. After standing for 3 hours, the supernatant was replaced with fresh acetone. This process was repeated 2 additional times. The powder was then isolated and thermally activated under dynamic vacuum on a Smart VacPrep $\left(65^{\circ} \mathrm{C}, 24 \mathrm{~h}\right)$. The recovered material, Cu-SIM-NU-901, was kept in a desiccator for storage. The $\mathrm{Cu}$ loading was determined to be $5.3 \mathrm{Cu}$ on each $\mathrm{Zr} 6$ node using ICP-OES.

Preparation of Cu-SIM-NU-907: $100 \mathrm{mg} \mathrm{Cu}(\mathrm{dmap})_{2}(0.373 \mathrm{mmol})$ was dissolved in $12 \mathrm{~mL}$ anhydrous heptane in Ar atmosphere in glovebox. $75 \mathrm{mg}(0.049 \mathrm{mmol})$ activated NU-907 were added to this solution and mixture was shaken until the MOFs disperse into solution. A color change from orange to brown was observed. The solution was allowed to stand at room temperature for overnight. The supernatant was removed and replaced with fresh anhydrous heptane $(12 \mathrm{~mL})$. Again the solution was shaken to disperse the MOF and allowed to stand for several hours (around $3 \mathrm{~h}$ ). This process was repeated 2 additional times. The supernatant was replaced with acetone upon removing from the glovebox. After standing for 3 hours, the supernatant was replaced with fresh acetone. This process was repeated 2 additional times. The powder was then isolated and thermally activated under dynamic vacuum on a Smart VacPrep $\left(150{ }^{\circ} \mathrm{C}, 18 \mathrm{~h}\right)$. The recovered material, Cu-SIM-NU-907, was kept in a desiccator for storage. The $\mathrm{Cu}$ loading was determined to be $1.9 \mathrm{Cu}$ on each $\mathrm{Zr} 6$ node using ICP-OES. 


\section{Optical Images of MOF Samples}
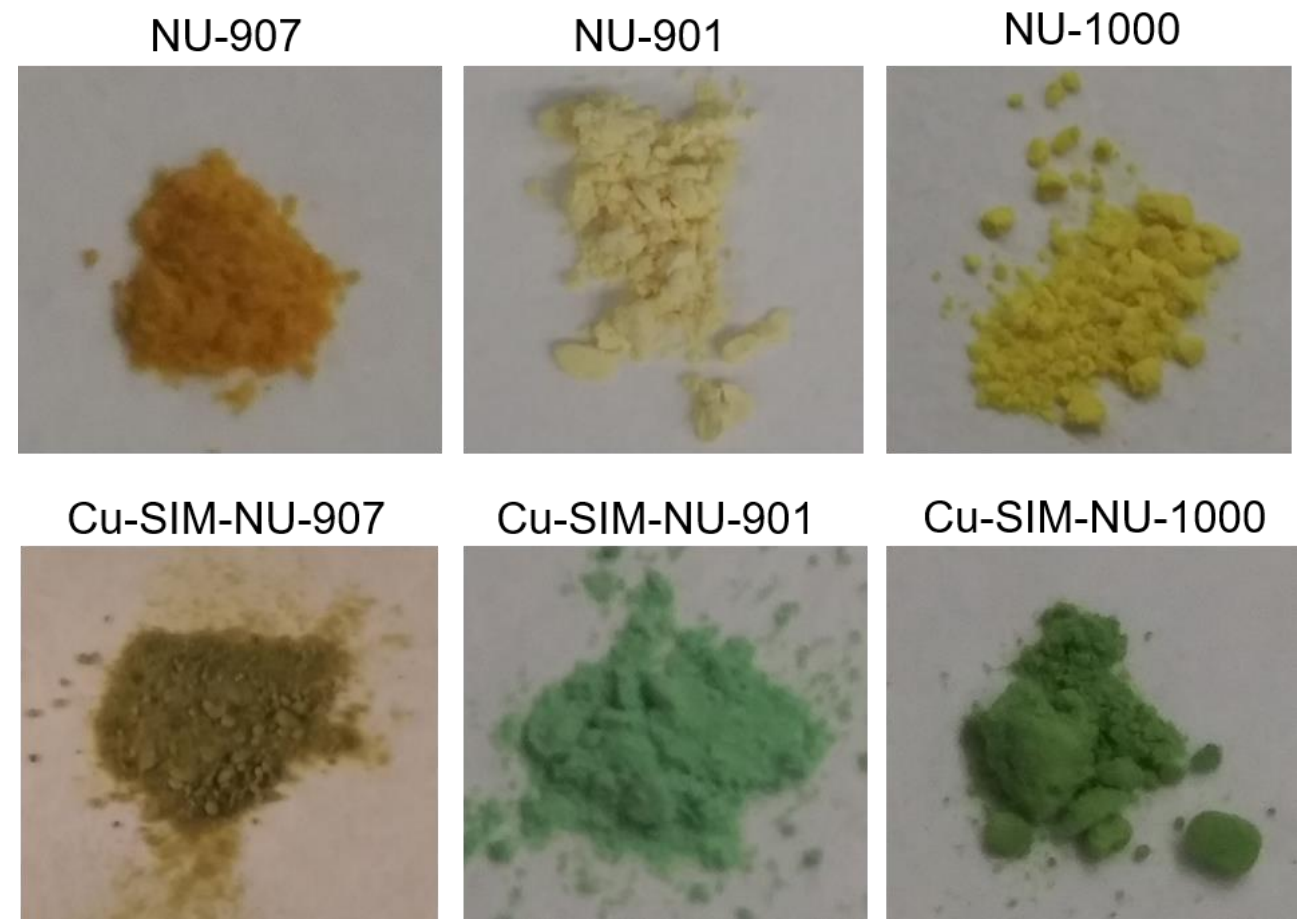

Cu-SIM-NU-1000

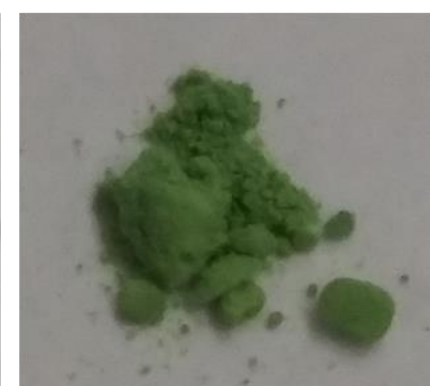

Figure S1. Photographs of MOF samples. NU-907, NU-901, and NU-1000 (top and from left to right). Cu-SIM-NU-907, Cu-SIM-NU-901, Cu-SIM-NU-1000 (Bottom and left to right) 


\section{$\underline{\text { X-ray Photoelectron Spectroscopy }}$}

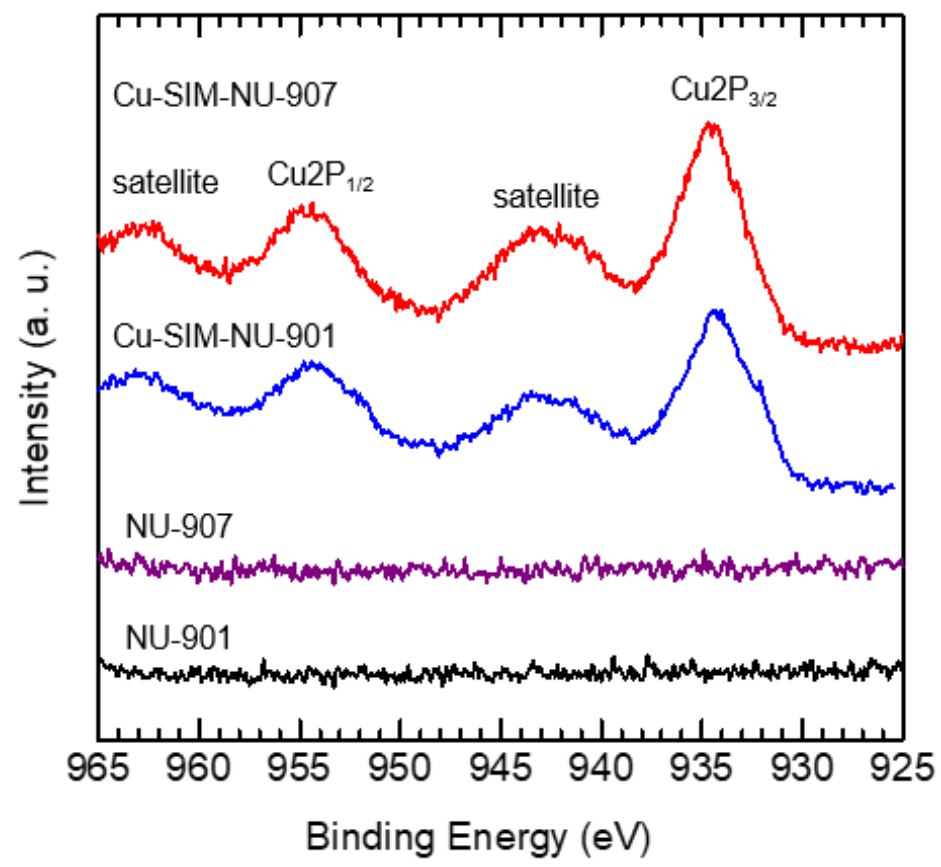

Figure S2. Cu2p XPS spectra for NU-901 (black), NU-907 (purple), Cu-SIM-NU901(blue) and Cu-SIM-NU-907 (red). The peak positions were normalized according to reference peak of $1 \mathrm{~s}$ carbon at $284.8 \mathrm{eV}$ 
PXRD Patterns for NU-901, Cu-SIM-NU-901 and Post-catalysis CuNPs@ NU-901

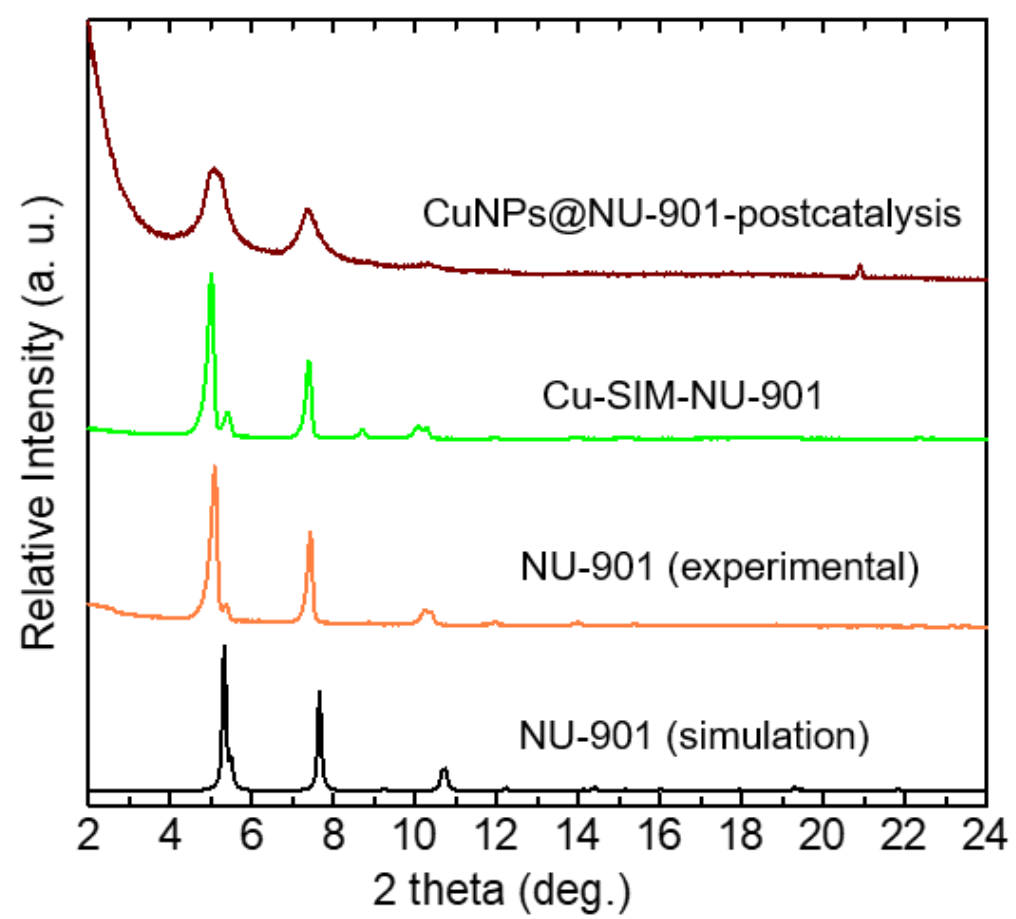

Figure S3. Powder X-ray diffraction patterns of NU-901 (orange), CU-SIM-NU901 (green) and CuNPs@NU-901-postcatalysis (brown). The small peak around $21^{\circ} 2 \theta$ in CuNPs@NU-901-postcatalysis is assigned to residual $\mathrm{SiO}_{2}$ from the catalyst bed support. 


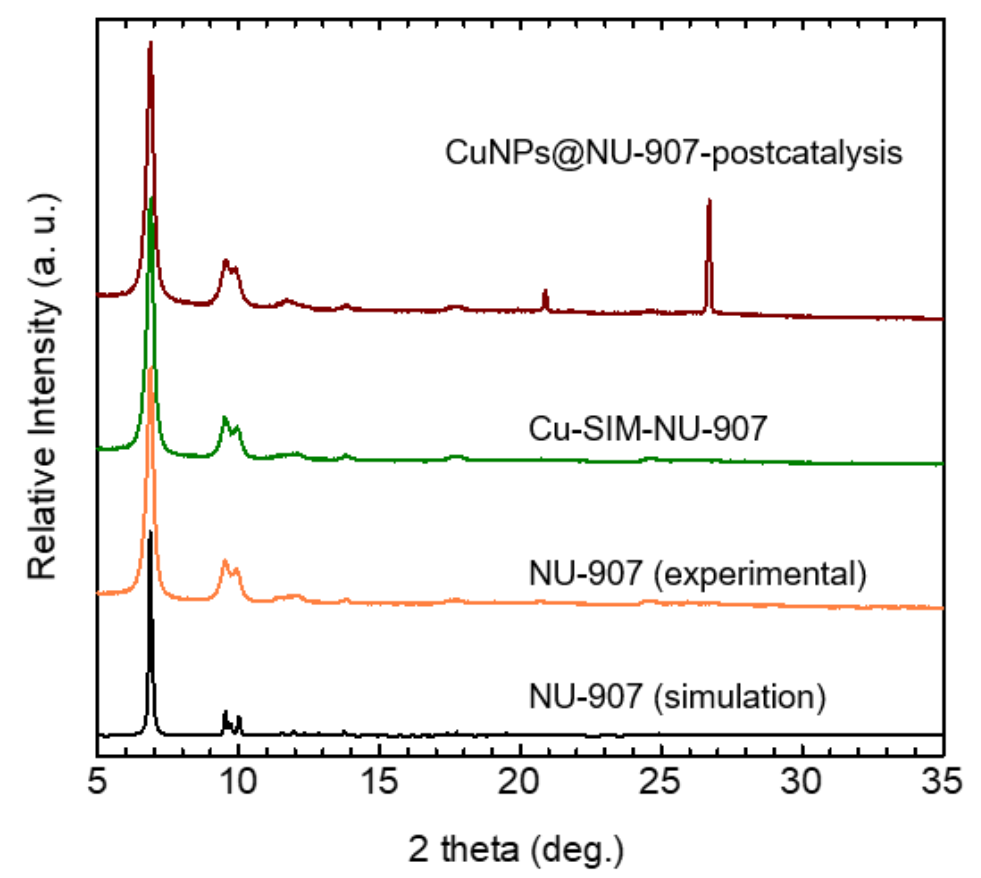

Figure S4. Powder X-ray diffraction patterns of NU-907 (orange), Cu-SIM-NU-907 (green) and CuNPs@NU-907-postcatalysis (brown). The additional peaks around $21^{\circ}$ and $27^{\circ} 2 \theta$ in CuNPs@NU-907-postcatalysis are assigned to residual $\mathrm{SiO}_{2}$ from the catalyst bed support. 
$\underline{\text { N2}_{2} \text { isotherms for NU-901, Cu-SIM-NU-901 and Post-catalysis CuNPs@NU-901 }}$

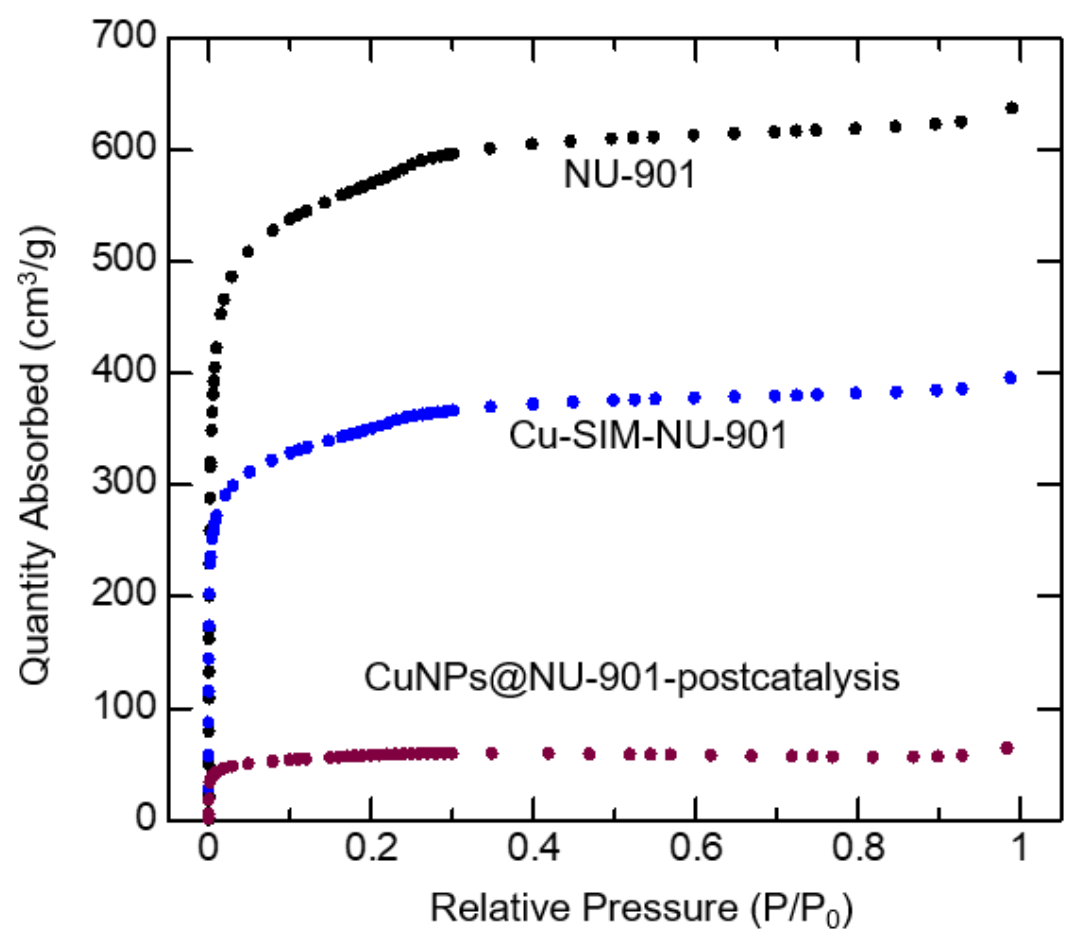

Figure S5. $\mathrm{N}_{2}$ isotherms of NU-901 (black), Cu-SIM-NU-901 (blue) and CuNPs@NU-901-postcatalysis (maroon) at $77 \mathrm{~K}$. 
$\underline{\text { N2}_{2} \text { isotherms for NU-907, Cu-SIM-NU-907 and Post-catalysis CuNPs@NU-907 }}$

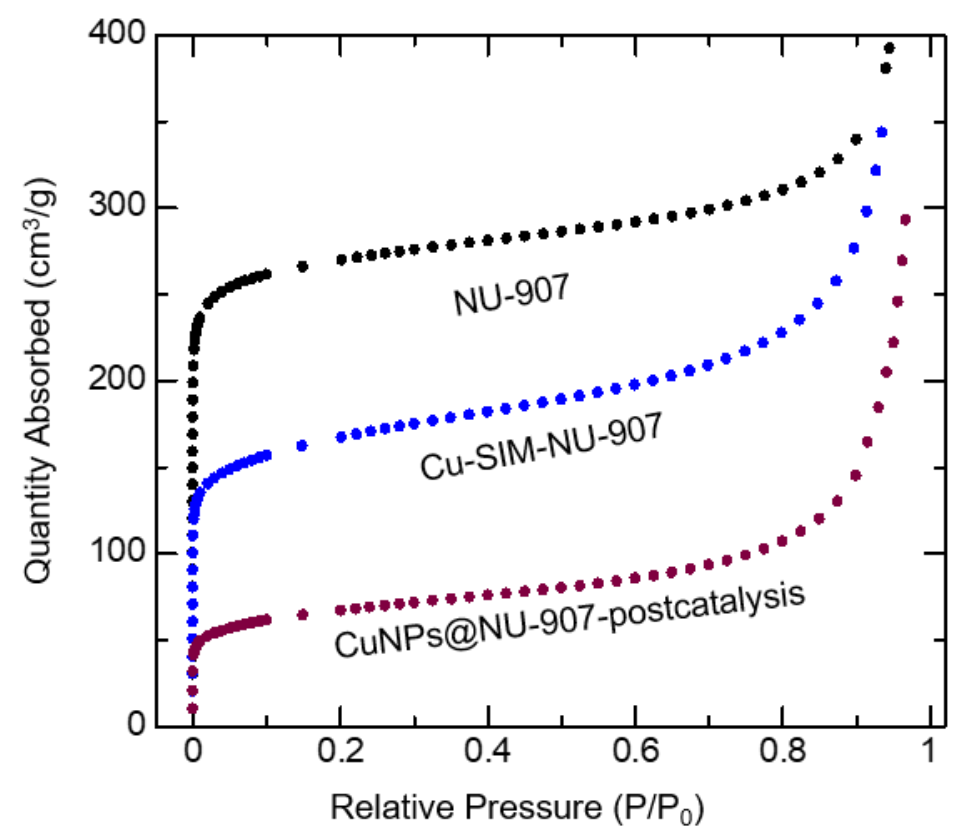

Figure S6. $\mathrm{N}_{2}$ isotherms of NU-907 (black), Cu-SIM-NU-907 (blue) and CuNPs@NU-907-postcatalysis (maroon) at $77 \mathrm{~K}$. 
Pore Size Distribution of NU-901, Cu-SIM-NU-901 and post-catalysis of CuNPs@NU-

$\underline{901}$

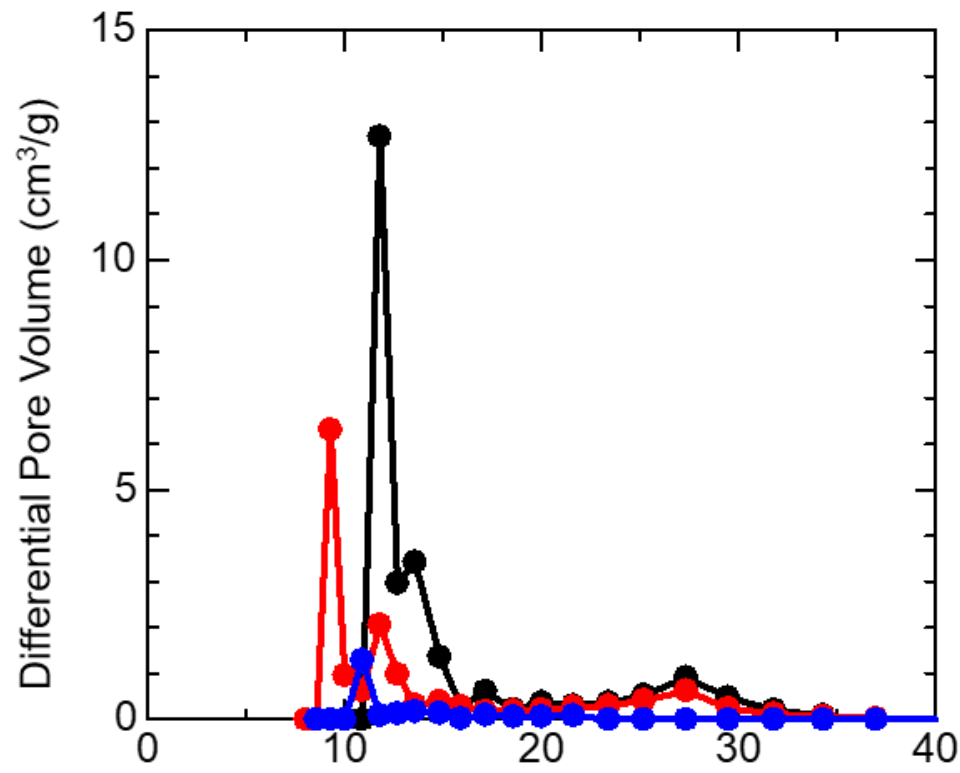

Figure S7. Pore size distribution for Width -901 (black), Cu-SIM-NU901 (red) and post catalysis of CuNPs@ NU-901 (blue). 
Pore Size Distribution of NU-907, Cu-SIM-NU-907 and post-catalysis of CuNPs@NU-

907

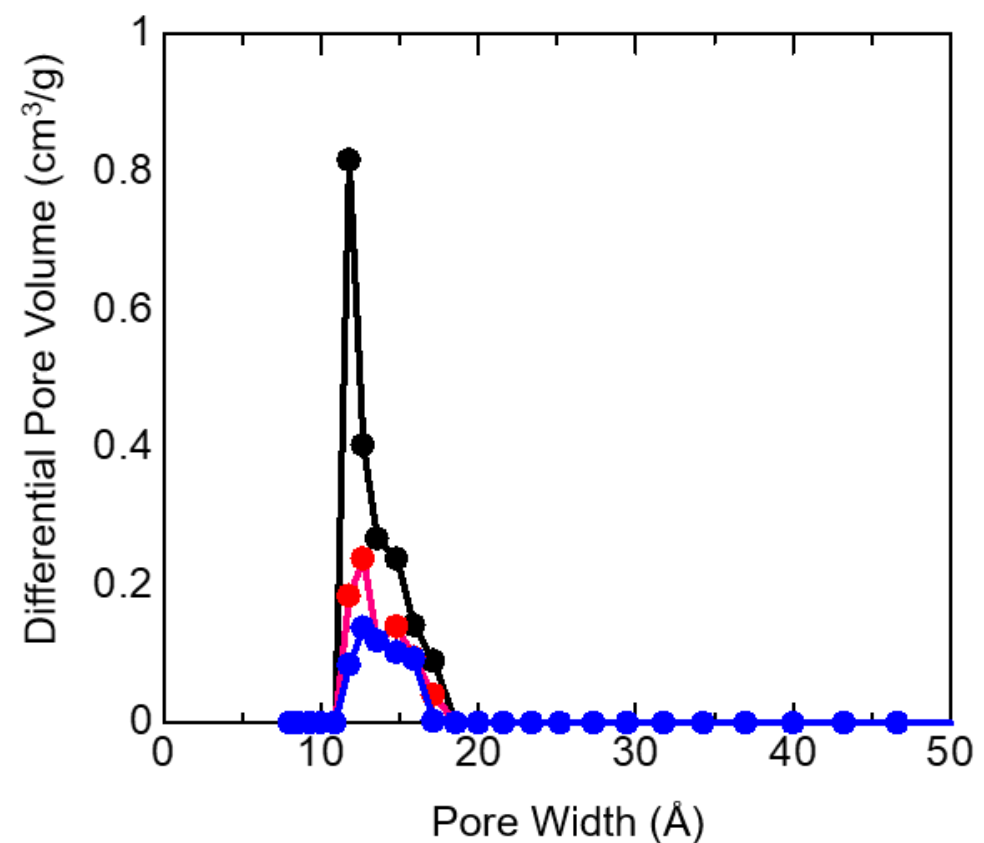

Figure S8. Pore size distributions for NU-907 (black), Cu-SIM-NU907 (red) and post catalysis of CuNPs@ NU-907 (blue). 

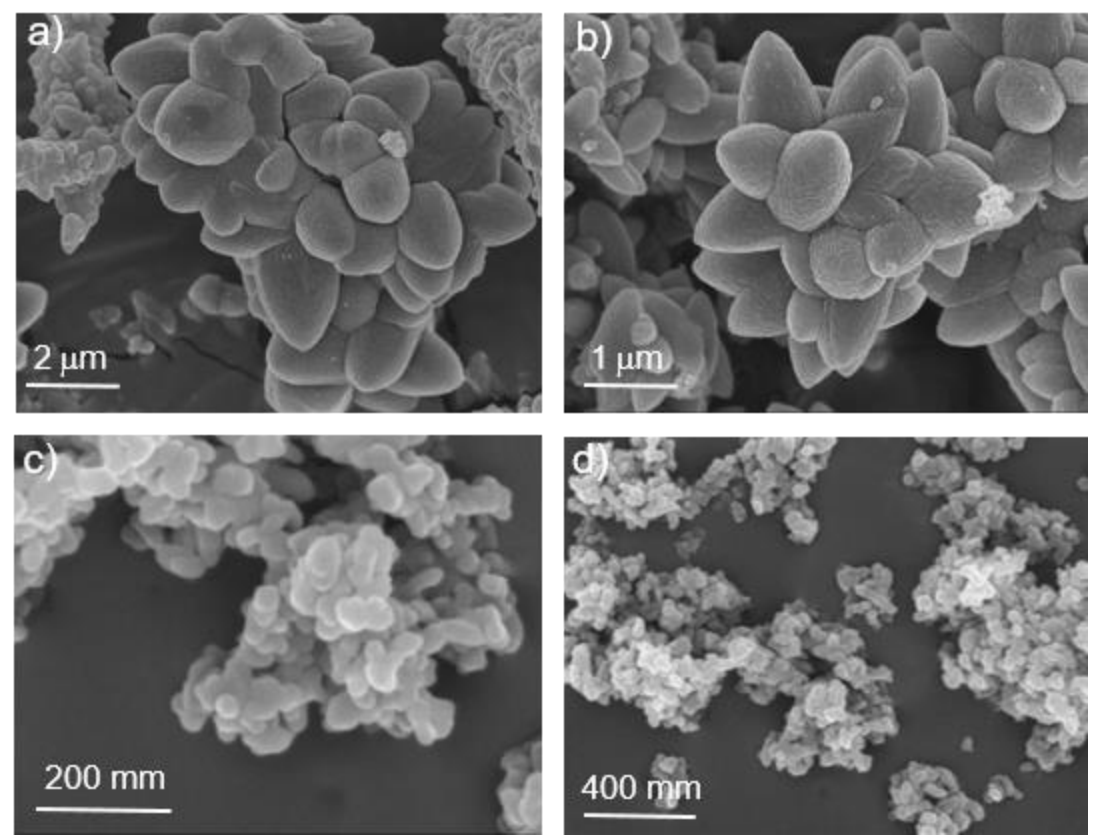

Figure S9. SEM images of (a) NU-901, (b) Cu-SIM-NU-901, (c) NU-907 and (d) Cu-SIM-NU-907 


\section{$\underline{\text { Computational Details }}$}

We have used periodic DFT calculations to model the semi hydrogenation reaction. The pathway for the formation of $\mathrm{C}_{2} \mathrm{H}_{4}$ from $\mathrm{C}_{2} \mathrm{H}_{2}$ were studied over $\mathrm{Cu}_{38}$ nanoparticle, $\mathrm{Cu}(100)$ and $\mathrm{Cu}(111)$ surfaces (Figure $\mathrm{S} 10$ ) that contains 38, 75 and $75 \mathrm{Cu}$ atoms, respectively. $\mathrm{Cu}(100)$ and $\mathrm{Cu}(111)$ surface was used as a model for the bigger nanoparticles. To model the reactions, first we explored different possible hydrogen absorption sites over both the nanoparticle and the surfaces. We found that hydrogen is preferentially absorbed on the bridging site for the $\mathrm{Cu} 38$ nanoparticle, while hollow and hcp sites are energetically most favorable for $\mathrm{Cu}(100)$ and $\mathrm{Cu}(111)$ surfaces, respectively (Figure S11). Similarly, different possible second hydrogen absorption sites are predicted with respect to the first hydrogen absorbed sites as shown in Figure S12. Our calculations indicate that the second hydrogen is efficiently absorbed at the bridge site for the $\mathrm{Cu}_{38}$ nanoparticle, at the hollow site for $\mathrm{Cu}(100)$ and hcp sites for $\mathrm{Cu}(111)$ surfaces, respectively. For the $\mathrm{Cu} 38$ nanoparticle there are two energetically favorable structures, namely the bridge-bridge-close and bridge-bridge-far, where the hydrogens remain close to each other and far from each other, respectively (Figure $\mathrm{S} 11)$. Next, we found different $\mathrm{C}_{2} \mathrm{H}_{2}$ absorption sites over the hydrogenated $\mathrm{Cu}$ nanoparticle and surfaces and the lowest energy structures have been further used to compute the reaction energies (Figure S13). 

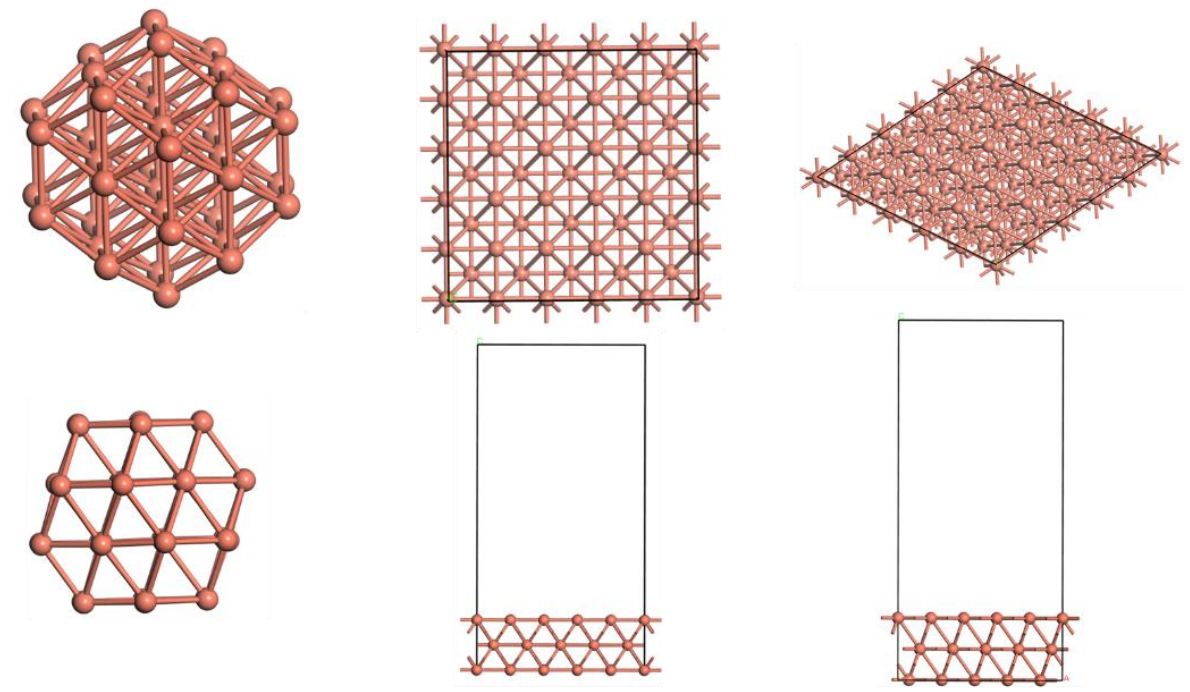

Cu 38

Cu 100

Cu 111

surface

Figure S10. Optimized structure of $\mathrm{Cu}_{38}$ nanoparticle and $\mathrm{Cu}(100)$ and $\mathrm{Cu}(111)$ surfaces, top view and side view.
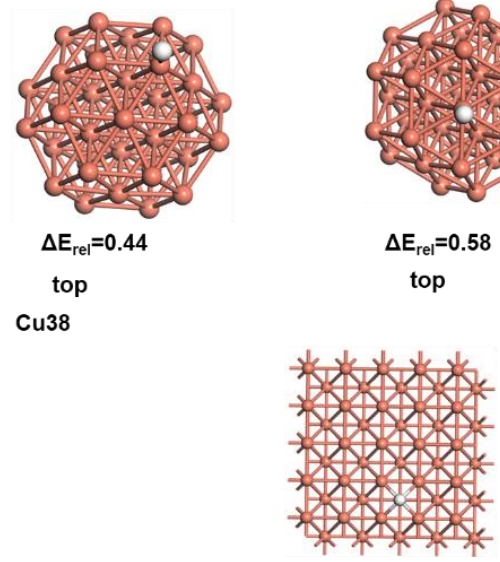

hollow

$\mathrm{Cu}(100)$

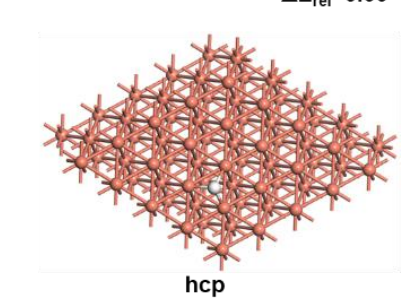

$\mathrm{Cu}(111)$

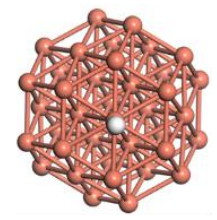

$\Delta \mathrm{E}_{\mathrm{rel}}=\mathbf{0 . 5 8}$

top
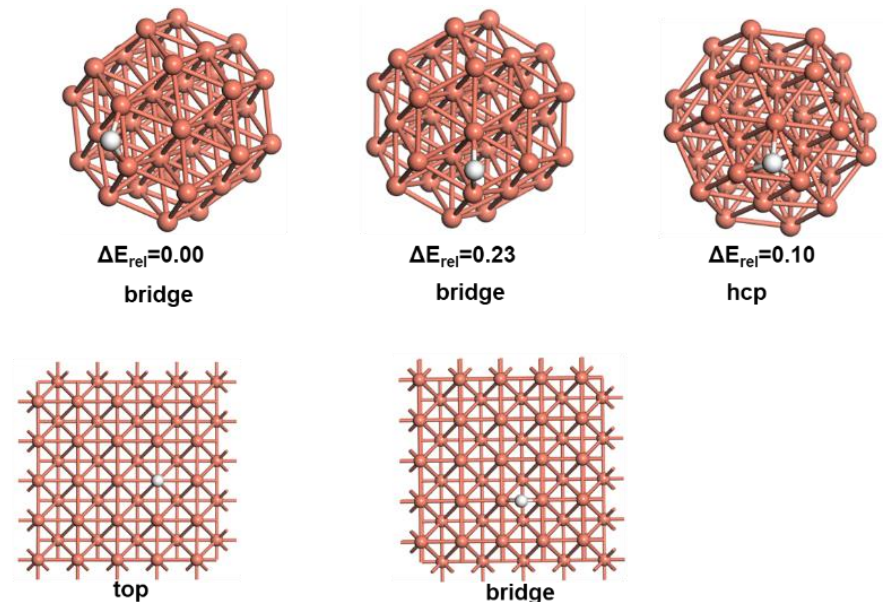

$\Delta \mathrm{E}_{\mathrm{rel}}=\mathbf{0 . 4 2}$

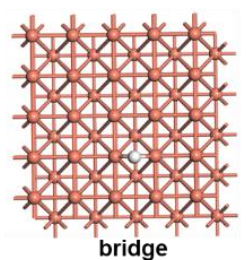

$\Delta \mathrm{E}_{\mathrm{rel}}=0.08$

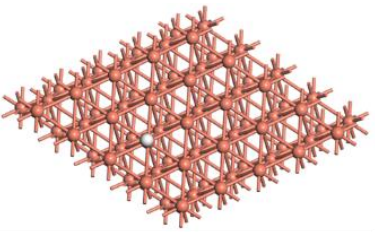

top

$\Delta \mathrm{E}_{\mathrm{rel}}=\mathbf{0 . 3 7}$

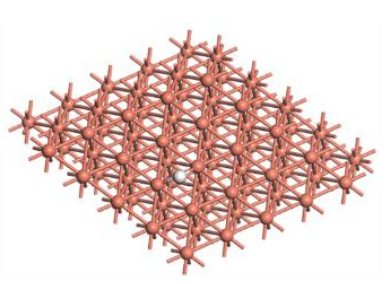

bridge

$\Delta \mathrm{E}_{\mathrm{rel}}=0.06$

Figure S11. Different possible hydrogen absorption sites for $\mathrm{Cu} 38$ nanoparticle, $\mathrm{Cu}(100)$ and $\mathrm{Cu}(111)$ surfaces. $\Delta \mathrm{E}_{\text {rel }}$ is the relative energy in $\mathrm{eV}$ with respect to the most stable structure. 


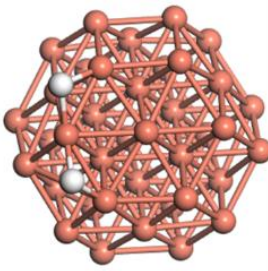

$\Delta \mathrm{E}_{\mathrm{rel}}=\mathbf{0 . 0 0}$

a(i)

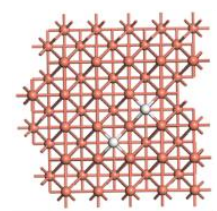

$\Delta \mathrm{E}_{\mathrm{rel}}=\mathbf{0 . 0 0}$

b(i)

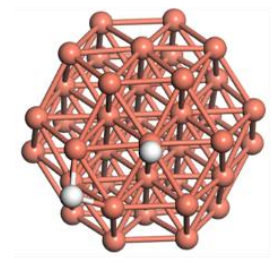

$\Delta \mathrm{E}_{\mathrm{rel}}=0.65$

a(ii)

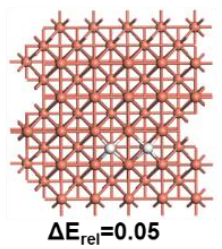

b(ii)

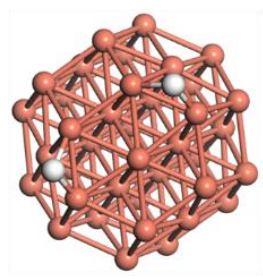

$\Delta \mathrm{E}_{\mathrm{rel}}=0.00$

a(iii)

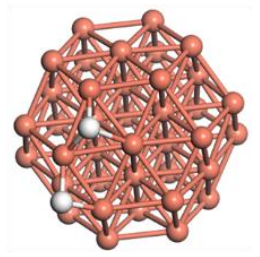

$\Delta \mathrm{E}_{\mathrm{rel}}=0.11$

a(iv)

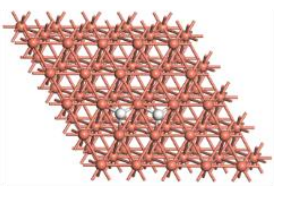

$\Delta \mathrm{E}_{\mathrm{rel}}=\mathbf{0 . 0 0}$

c(i)

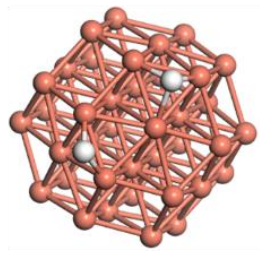

$\Delta E_{r e l}=0.13$

$a(v)$

Figure S12. Different possible second hydrogen absorption sites for (a) $\mathrm{Cu}_{38}$ nanoparticle, (b) $\mathrm{Cu}(100)$ and (c) $\mathrm{Cu}(111)$ surfaces with respect to the first hydrogen absorbed structure. $\Delta \mathrm{E}_{\text {rel }}$ is the relative energy in $\mathrm{eV}$ with respect to the most stable structure. Herein, a(i) bridgebridge-close, a(ii) bridge-top, a(iii) bridge-bridge-far, a(iv) bridge-hcp-close, a(v) bridgehcp-far, b(i) hollow-hollow-close, b(i) hollow-hollow-far, c(i) hcp-hcp-close and c(i) hcphcp-far.

(a) Cu 100 Surface 1x-1
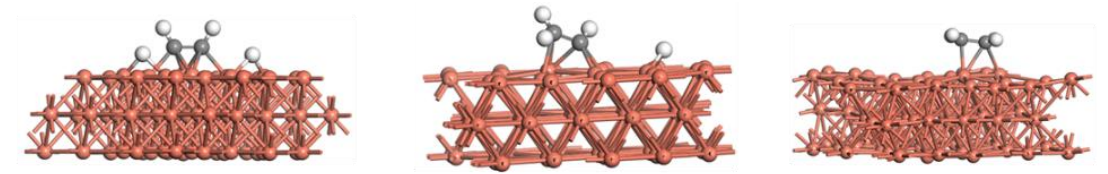

(b) Cu 111 Surface

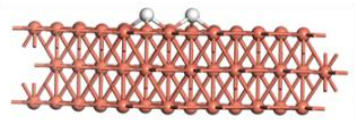

*2H

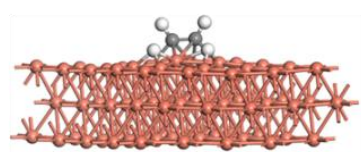

$* 2 \mathrm{H}-\mathrm{C}_{2} \mathrm{H}_{2}$

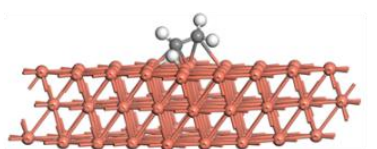

${ }^{*} \mathrm{H}-\mathrm{C}_{2} \mathrm{H}_{3}$

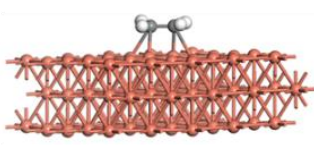

${ }^{*} \mathrm{C}_{2} \mathrm{H}_{4}$

Figure S13. Optimized geometries during the hydrogenation of acetylene to ethylene over (a) $\mathrm{Cu}(100)$ and (b) $\mathrm{Cu}(111)$ surfaces. 


\section{$\underline{\text { Representative Gas Chromatograms }}$}

a)

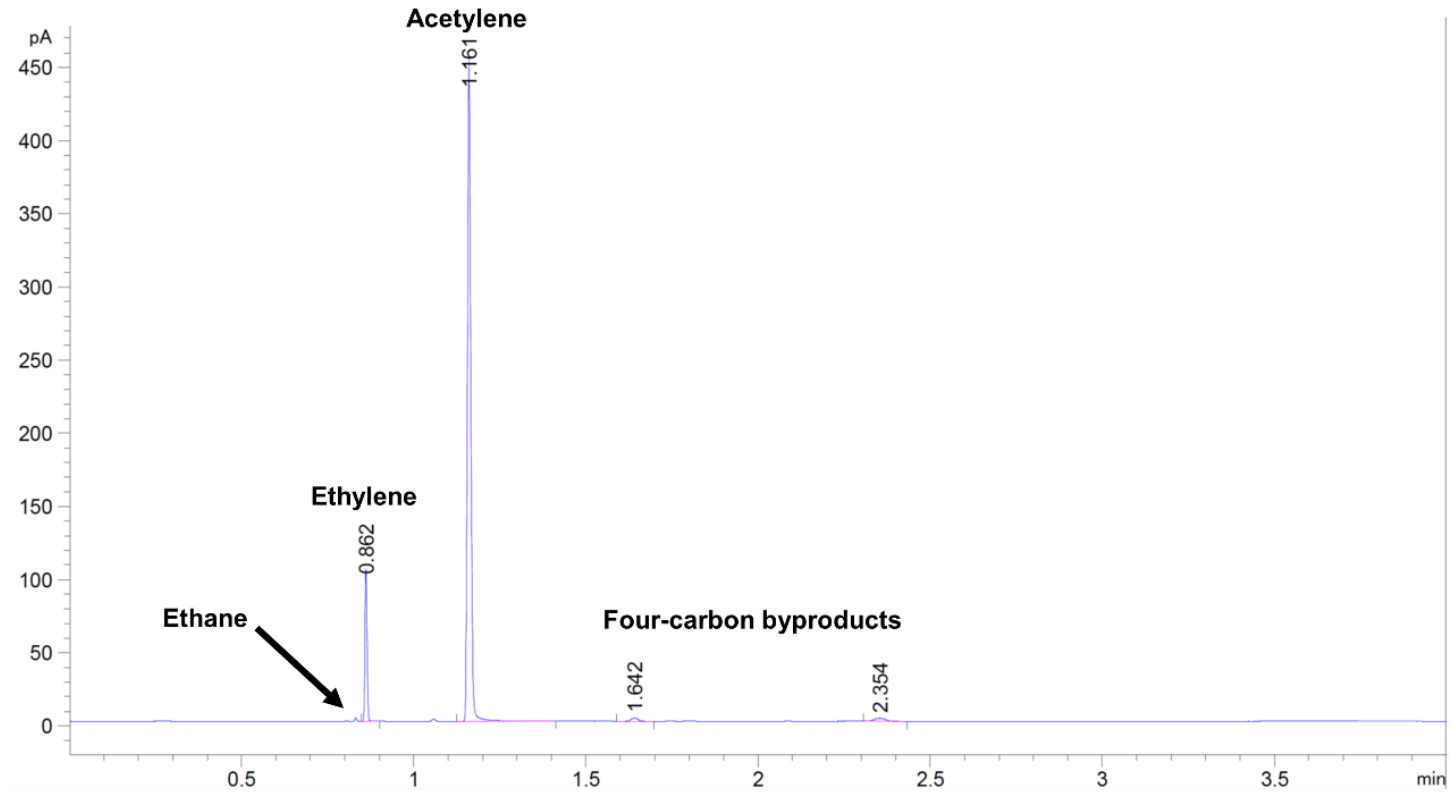

b)

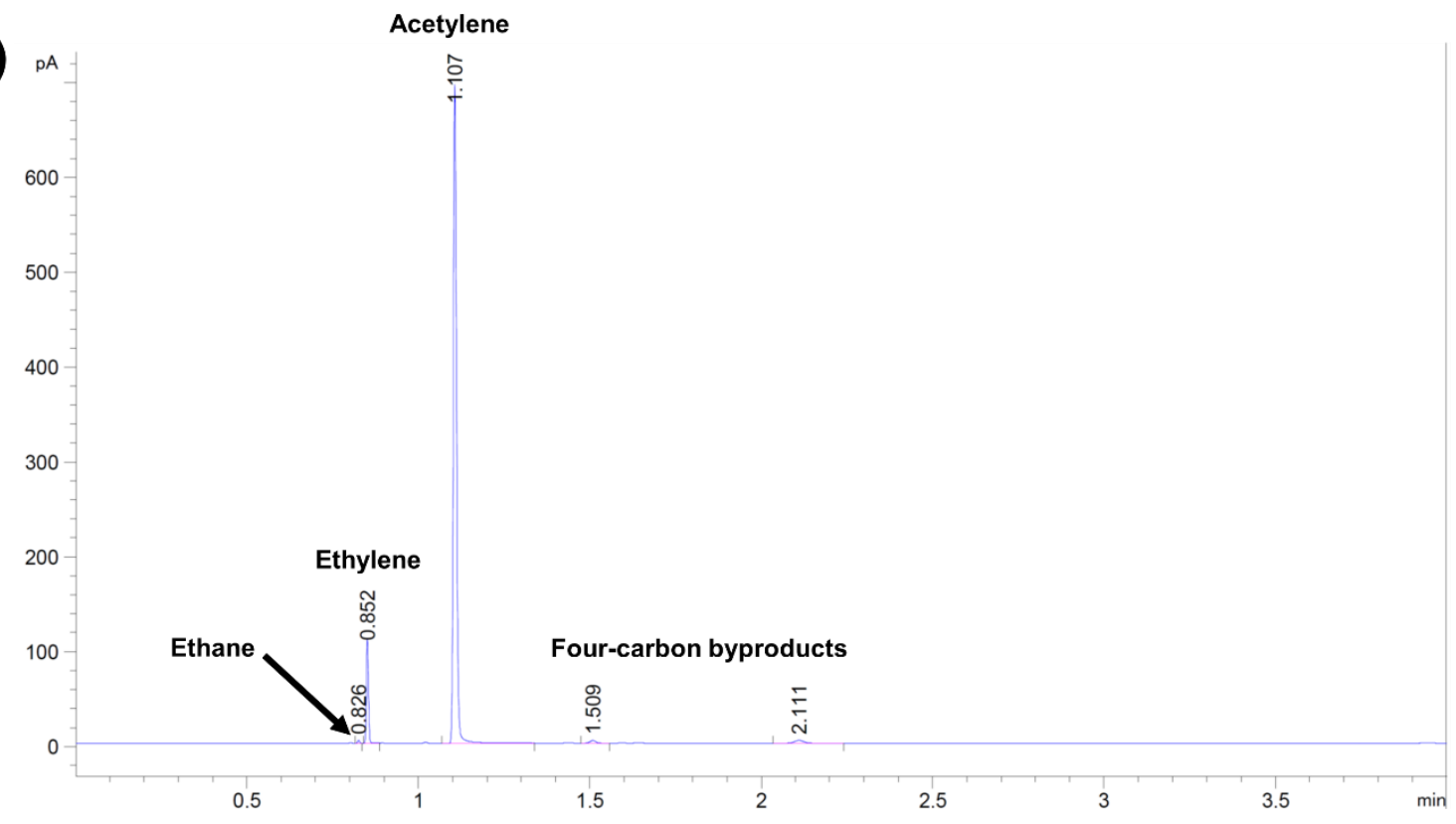

Figure S14. Representative gas chromatograms for catalysis products of (a) CuNPs@NU-901 and (b) CuNPs@NU-907. 


\section{Diffusion limit test for CuNPs@NU-901}

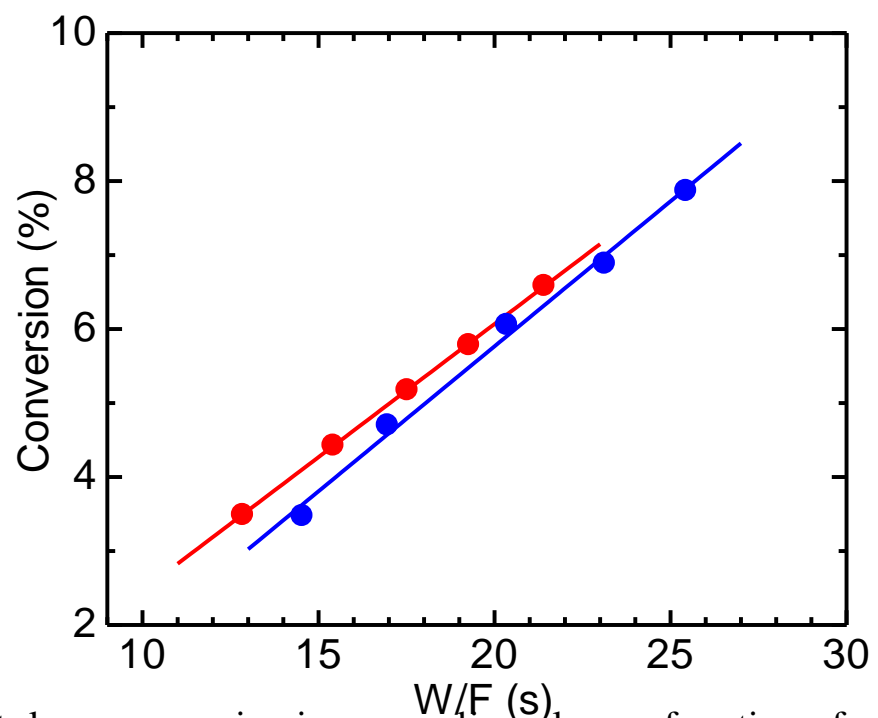

Figure S15. Acetylene conversion increases linearly as a function of contact time for CuNPs@NU 901. The close agreement of two samples with different catalyst masses (red and blue) indicates that the process is not diffusion limited.

\section{Diffusion limit test for CuNPs@NU-907}

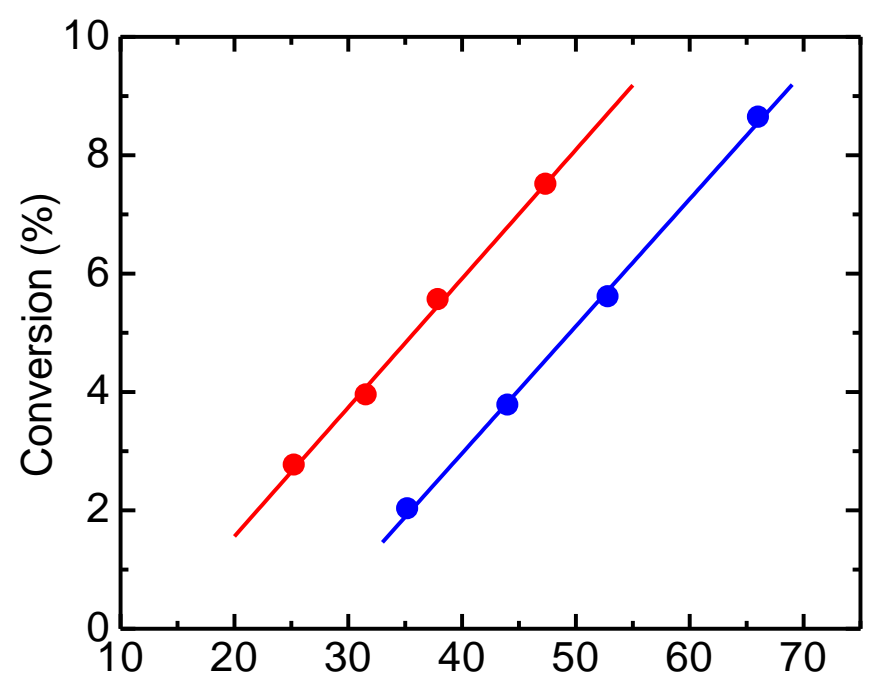

Figure S16. Acetylene conversion increa 1 /Fipgarly as a function of contact time for CuNPs@NU-907. The close agreement of two samples with different catalyst masses (red and blue) indicates that the process is not diffusion limited. 


\section{References}

(1) Toby, B. H.; Von Dreele, R. B., GSAS-II: the genesis of a modern open-source all purpose crystallography software package. J. Appl. Crystallogr. 2013, 46, 544-549.

(2) Chupas, P. J.; Chapman, K. W.; Kurtz, C.; Hanson, J. C.; Lee, P. L.; Grey, C. P., A versatile sample environment cell for non-ambient X-ray scattering experiments. J. Appl. Crystallogr. 2008, 41, 822824.

(3) Le Bail, A., Modelling the silica glass structure by the Rietveld method. J. Non-Cryst. Solids 1995, 183, 39-42.

(4) Petříček, V.; Dušek, M.; Palatinus, L., Crystallographic Computing System JANA2006: General features. In Zeitschrift für Kristallographie - Crystalline Materials, 2014; Vol. 229, p 345.

(5) Yakovenko, A. A.; Wei, Z.; Wriedt, M.; Li, J.-R.; Halder, G. J.; Zhou, H.-C., Study of Guest Molecules in Metal-Organic Frameworks by Powder X-ray Diffraction: Analysis of Difference Envelope Density. Cryst. Growth Des. 2014, 14, 5397-5407.

(6) Qiu, X.; Thompson, J. W.; Billinge, S. J. L., PDFgetX2: a GUI-driven program to obtain the pair distribution function from X-ray powder diffraction data. J. Appl. Crystallogr. 2004, 37, 678.

(7) Farrow, C. L.; Juhas, P.; Liu, J. W.; Bryndin, D.; Božin, E. S.; Bloch, J.; Th, P.; Billinge, S. J. L., PDFfit2 and PDFgui: computer programs for studying nanostructure in crystals. J. Phys.: Condens. Matter 2007, 19, 335219.

(8) Chen, Z.; Islamoglu, T.; Farha, O. K., Toward Base Heterogenization: A Zirconium Metal-Organic Framework/Dendrimer or Polymer Mixture for Rapid Hydrolysis of a Nerve-Agent Simulant. ACS Applied Nano Materials 2019, 2, 1005-1008.

(9) Wang, H.; Dong, X.; Lin, J.; Teat, S. J.; Jensen, S.; Cure, J.; Alexandrov, E. V.; Xia, Q.; Tan, K.; Wang, Q.; Olson, D. H.; Proserpio, D. M.; Chabal, Y. J.; Thonhauser, T.; Sun, J.; Han, Y.; Li, J., Topologically guided tuning of $\mathrm{Zr}$-MOF pore structures for highly selective separation of $\mathrm{C} 6$ alkane isomers. Nat. Commun. 2018, 9, 1745. 